\title{
Anatomic fit of precontoured extra-articular distal humeral locking plates: a cadaveric study
}

\author{
Joon-Ryul Lim ${ }^{1}$, Tae-Hwan Yoon ${ }^{1}$, Hwan-Mo Lee ${ }^{2}$, Yong-Min Chun ${ }^{1}$ \\ ${ }^{1}$ Department of Orthopedic Surgery, Arthroscopy and Joint Research Institute, Severance Hospital, Yonsei University College of Medicine, Seoul, Korea \\ ${ }^{2}$ Department of Orthopedic Surgery, Severance Hospital, Yonsei University College of Medicine, Seoul, Korea
}

\begin{abstract}
Background: Extra-articular distal humerus locking plates (EADHPs) are precontoured anatomical plates widely used to repair distal humeral extra-articular diaphyseal fractures. However, EADHPs frequently cause distal protrusion and resulting skin discomfort. The purpose of this study was to predict the occurrence of anatomic fit mismatch. We hypothesized that the smaller the humerus size, the greater the anatomic fit mismatch with EADHP.

Methods: Twenty humeri were analyzed in this study. Humeral length and distal humeral width were used as parameters of humeral size. Plate protrusion was measured between the EADHP distal tip and the distal humerus. We set the level of unacceptable EADHP anatomic fit mismatch as $\geq 10 \mathrm{~mm}$ plate protrusion.

Results: A significant negative linear correlation was also confirmed between humeral size and plate protrusion, with a coefficient of determination of 0.477 for humeral length and 0.814 for distal humeral width. The cutoff value of humeral length to avoid $\geq 10 \mathrm{~mm}$ plate protrusion was $293.6 \mathrm{~mm}$ (sensitivity, $88.9 \%$; specificity, $81.8 \%$ ) and for distal humeral width was $60.5 \mathrm{~mm}$ (sensitivity, 100\%; specificity, $81.8 \%$ ). Conclusions: Anatomic fit mismatch in distal humeral fractures after EADHP fixation has a negative linear correlation with humeral length and distal humeral width. For patients with a distal humeral width $<60.5 \mathrm{~mm}, \geq 10 \mathrm{~mm}$ plate protrusion will occur when an EADHP is used, and an alternative implant or approach should be considered.
\end{abstract}

Keywords: Bone plates; Humeral fractures; Prostheses and implants; Humerus

\section{INTRODUCTION}

Distal-third humeral fractures account for up to $2 \%$ of all adult fractures [1], and they are challenging to surgically correct [2-4]. Various anatomical precontoured locking plates have recently been developed and are used for surgical treatment of distal humerus fractures [5-7]. Extra-articular distal humerus locking plates (EADHPs; DePuy Synthes, Oberdorf, Switzerland) are anatomical precontoured plates widely used in distal humeral extra-articular diaphyseal fractures [8-12].

The posterolateral elbow column is used to fix the EADHP with a posterior approach. Despite the distally tapered design of the plate, it causes plate protrusion and skin discomfort after surgery (Fig. 1). Implant prominence after EADHP fixation was

\footnotetext{
Received: April 7, $2021 \quad$ Revised: April 26, $2021 \quad$ Accepted: April 28, 2021

Correspondence to: Yong-Min Chun

Department of Orthopedic Surgery, Arthroscopy and Joint Research Institute, Severance Hospital, Yonsei University College of Medicine, 50-1 Yonseiro, Seodaemun-gu, Seoul 03722, Korea

Tel: +82-2-2228-5679, Fax: +82-2-363-6248, E-mail: min1201@hanmail.net, ORCID: https://orcid.org/0000-0002-8147-6136
}

\section{Financial support: None.}

Conflict of interest: None. 
noted in up to $59.5 \%$ of cases [13]. This problem requires implant removal after fracture union $[11,12]$. However, in most distal humeral fracture cases, EADHPs should be placed beneath the radial nerve (Fig. 2). Thus, iatrogenic radial nerve palsy is likely to result from EADHP removal surgery.

Zhou et al. [14] reported that EADHP caused approximately $8^{\circ}$ of anatomic fit mismatch in the shafts of adult Chinese bodies in a cadaveric humeri study, which can be resolved by bending the plate. However, anatomic fit mismatch with plate protrusion oc-

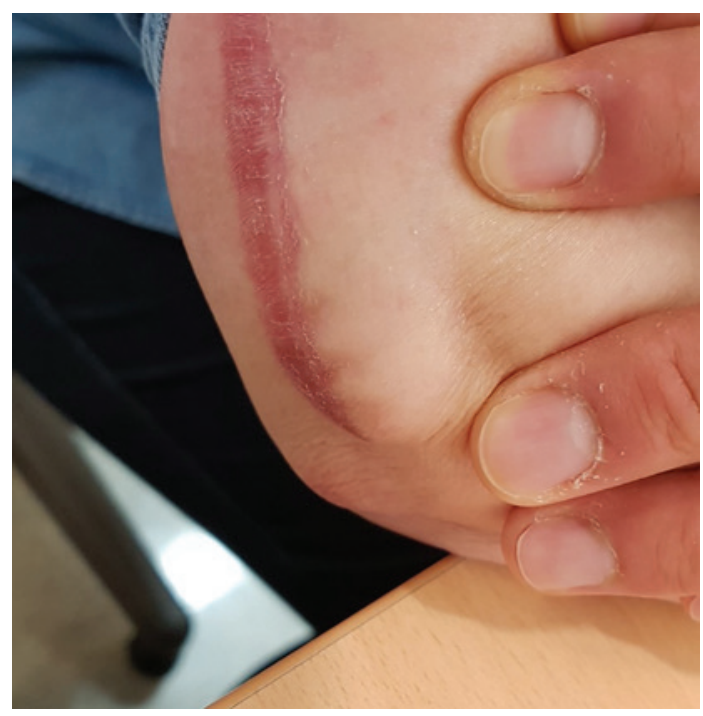

Fig. 1. Skin prominence after application of a precontoured extra-articular distal humeral locking plate.

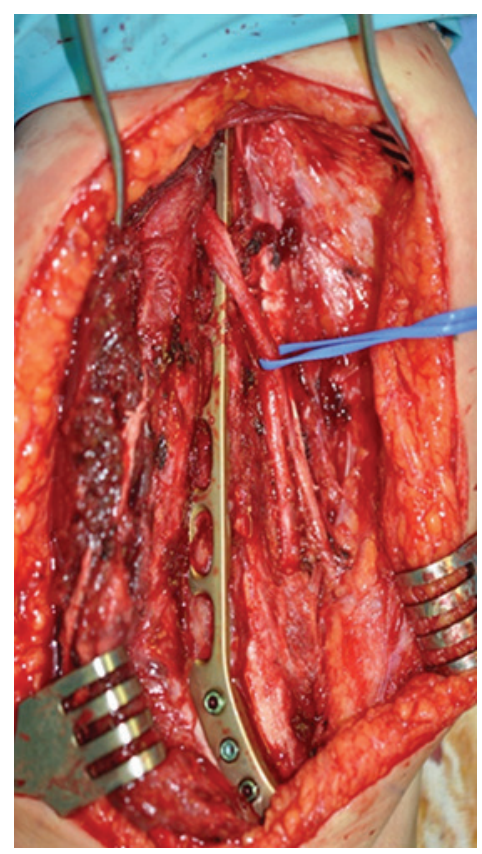

Fig. 2. Most extra-articular distal humerus locking plates are placed beneath the radial curring at the EADHP distal tip is likely to occur when the humerus is small, and anatomic fit mismatch is difficult to resolve through plate bending. Therefore, it is necessary to predict the plate protrusion occurrence and resulting skin discomfort that leads to risk of iatrogenic radial nerve palsy to better inform treatment planning and implant selection. However, the humeral size at which EADHP distal tip protrusion occurs due to anatomic fit mismatch has not been established.

The purpose of this study was to determine the humeral size cutoff for plate protrusion despite proper plate positioning through a cadaveric study. We hypothesized that the smaller the size of the humerus, the greater the anatomic fit mismatch of EADHP that would occur, and that the relationship between humeral size and mismatch will have a negative linear correlation.

\section{METHODS}

Because this study is a cadaveric study, there is no Institutional Review Board approval and informed consent for this study.

\section{Specimens}

A total of 20 humeri of various sizes were used. All soft tissue was removed, and the lack of any gross deformities of the humerus was confirmed. Humeral length and distal humeral width were used as parameters of humeral size. Humeral length was measured along the anatomical axis, and the distance between the humeral head tip and the trochlear tip with a perpendicular line on the axis was measured using digital tape (BL-DM; Bluetec, Daejeon, Korea). Distal humeral width was measured as the medial-to-lateral length between the medial and lateral epicondyles along the perpendicular plane of the anatomical axis using a digital caliper (SD500-150PRO; Sincon, Busan, Korea) (Fig. 3).

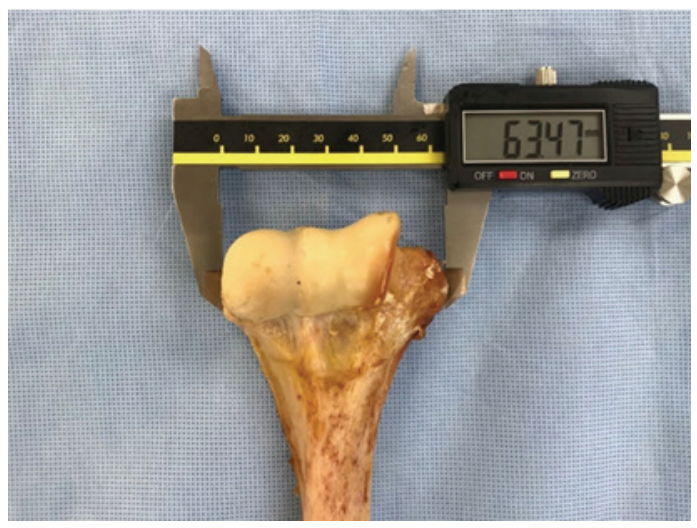

Fig. 3. Distal humeral width was measured using digital caliper between the medial epicondyle and the lateral epicondyle. 


\section{Measurement of Anatomic Fit Mismatch}

A six-hole EADHP was positioned sufficiently laterally to not encroach on the olecranon fossa. EADHP mismatch caused by humeral posterior angulation in the shaft area as well as in the distal part was due to the plate's distal five holes being longer than that of the distal humeral posterolateral column. The shaft mismatch could be resolved through proper EADHP bending [14], so the middle portion of the EADHP was bent using a plate bending press (Plate Bending Press 329.3; DePuy Synthes) to fit the contour of posterior angulation of the humerus. Additionally, mismatch occurring at the plate distal tip may be improved by proximal plate positioning, but in such cases, plate-bone mismatches occurred at the posterolateral column even if the plate was modified by the plate bending press. Therefore, we first fitted the EADHP to the posterolateral column, the mismatch occurring in the humeral shaft was resolved by the plate bending press, and the mismatch occurring in the plate distal tip was measured. The amount of mismatch between the plate and distal humerus was assessed by measuring the distance between the center point of the EADHP distal tip and the distal humeral bone point. The distal humeral bone point was set as a perpendicular line drawn from the plate distal tip center point to the humeral bone (Fig. 4). There have been no previous studies of EADHP anatomic fit mismatch and symptom occurrence. We defined unacceptable EADHP anatomic fit mismatch as plate protrusion $\geq 10 \mathrm{~mm}$.

\section{Statistical Analysis}

Simple linear regression was performed to estimate how humeral length and humoral width predicted protrusion distance. Receiver operating characteristic curve analysis was used to determine the appropriate cutoff value for plate protrusion, and the value with the largest Youden index (J) was defined as the optimal cutoff value [15]. Statistical power was set to 0.9 and the threshold

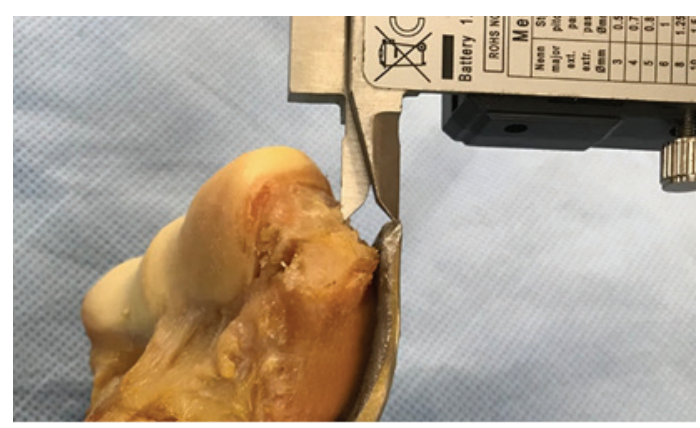

Fig. 4. The distal humeral bone point was set based on a perpendicular line drawn from the center point of the extra-articular distal humerus locking plate (EADHP) tip to the humeral bone. Plate protrusion was measured between the center point of the EADHP distal tip and the distal humeral bone point. for significance was set to $\mathrm{p} \geq 0.05$. All statistical analyses and tests were performed using IBM SPSS ver. 25.0 (IBM Corp., Armonk, NY, USA).

\section{RESULTS}

Mean humeral length was $301.13 \pm 23.3 \mathrm{~mm}$ and mean distal humeral width was $60.9 \pm 5.0 \mathrm{~mm}$. Mean plate protrusion distance was $9.1 \pm 2.7 \mathrm{~mm}$. In total, $45 \%(9 / 20)$ of humeri showed $\geq 10$ $\mathrm{mm}$ plate protrusion. A significant linear correlation was observed between humeral length and plate protrusion $(\mathrm{p}=0.001)$ and the coefficient of determination value $\left(R^{2}\right)$ was 0.477 . The best-fit linear equation was $\mathrm{Y}=32.85-0.08 \mathrm{X}$ (Fig. 5A). A significant linear correlation was also confirmed between distal humeral width and plate protrusion $(\mathrm{p}<0.001)$, and the $\mathrm{R}^{2}$ was 0.814 and the best-fit linear equation was $\mathrm{Y}=38.57-0.48 \mathrm{X}$ (Fig. 5B).

The area under the curve for humeral length was 0.879 and for distal humeral width was 0.944 . The maximal J value for humeral length was 0.707 ; thus, the cutoff value for humeral length to avoid $\geq 10 \mathrm{~mm}$ plate protrusion was $293.6 \mathrm{~mm}$ (sensitivity,
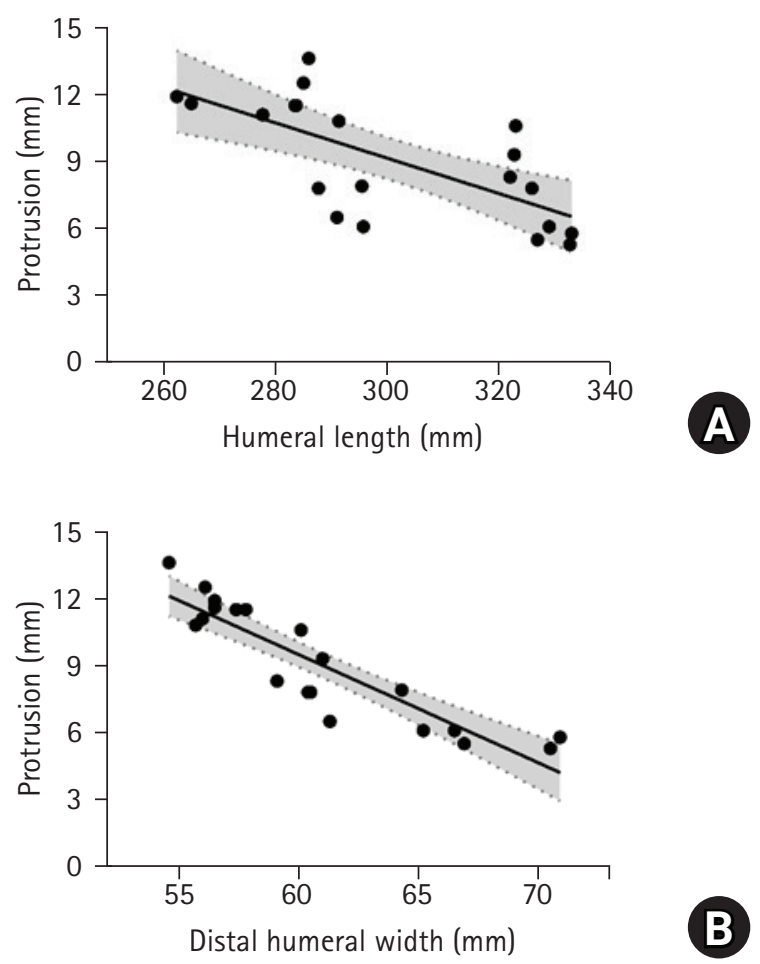

B

Fig. 5. (A) Scattergram of distal plate protrusion for humeral length the best-fit linear equation is calculated as $\mathrm{Y}=32.85-0.08 \mathrm{X}$, where $\mathrm{Y}$ represents the protrusion and $\mathrm{X}$ represents the humeral length. (B) Scattergram of distal plate protrusion for distal humeral width: the best-fit linear equation is calculated as $\mathrm{Y}=38.57-0.48 \mathrm{X}$, where $\mathrm{Y}$ represents the protrusion and $\mathrm{X}$ represents the distal humeral width. 


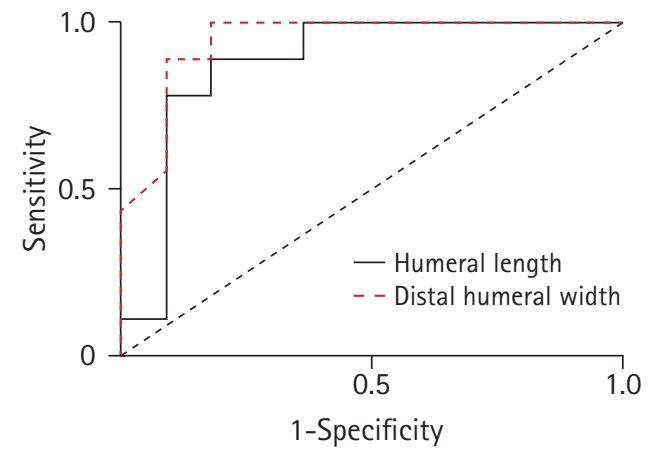

Fig. 6. Receiver operative characteristic curve analysis. The area under the curve for humeral length, $0.879 ; 90 \%$ confidence interval (CI), 0.74-1.0; $\mathrm{p}=0.004$. The cutoff value was $293.6 \mathrm{~mm}$ for humeral length, which corresponds to a sensitivity of $88.9 \%$ and a specificity of $81.8 \%$. The area under the curve for distal humeral width, 0.944 ; $90 \%$ CI, 0.86-1.0; $\mathrm{p}=0.001$. The cutoff value was $60.5 \mathrm{~mm}$ for distal humeral width, which corresponds to a sensitivity of $100 \%$ and a specificity of $81.8 \%$.

88.9\%; specificity, $81.8 \%$ ). The maximal J value for distal humeral width was 0.818 , and the cutoff value for humeral width was 60.5 mm (sensitivity, 100\%; specificity, 81.8\%) (Fig. 6).

\section{DISCUSSION}

The purpose of this study was to investigate whether anatomic fit mismatch between EADHP and the distal humerus increases as humeral size decreases when an EADHP is used to repair a distal humeral fracture. EADHP protrusion decreased by $0.08 \mathrm{~mm}$ per 1 - $\mathrm{mm}$ increase in humeral length. As distal humeral width increased by $1 \mathrm{~mm}$, plate protrusion decreased by $0.48 \mathrm{~mm}$. Additionally, when humeral length was $<293.6 \mathrm{~mm}$ or distal humeral width was $<60.5 \mathrm{~mm}$, plate protrusion was at least $10 \mathrm{~mm}$ at the distal humerus.

Despite the tapered design of the EADHP for the distal humerus, anatomic fit mismatch frequently occurred at the distal tip of the EADHP. The EADHP was designed to allow distal fixation with five locking screws (three bicortical screws proximally on the lateral column and two unicortical screws at the end toward the capitellum and trochlea) [11]. If EADHPs are proximally fixed to avoid distal tip protrusion in small-sized humeri, mismatch at the distal tip of the plate can be reduced to some degree, but a gap between the bone and plate occurs at the lateral column where the three bicortical screws are supposed to be fixed. If the gap is greater than $5 \mathrm{~mm}$, biomechanical stability is significantly decreased [16]. Therefore, these mismatches should be considered in preoperative planning.

Humeral length and distal humeral width were used as parameters for humeral size in this study. Distal humeral width was the most relevant measure, and it had approximately twice the $\mathrm{R}^{2}$ value of humerus length. In a previous cadaveric humeral estimation study, the $\mathrm{R}^{2}$ between the distance from the olecranon fossa upper margin to the trochlear tip and humeral length was 0.47 [17]. This $\mathrm{R}^{2}$ suggests that humeral length is not highly predictive of the size of the distal humeral region. Additionally, the $\mathrm{R}^{2}$ between humeral length and plate protrusion as measured in this study was 0.477 , which was similar to previous values observed between the distal humerus region and humeral length. Interestingly, the $\mathrm{R}^{2}$ between distal humeral width and protrusion was 0.814 , suggesting that distal humeral width is a better predictor of plate protrusion than humeral length.

Zhou et al. [14] reported a mismatch issue for EADHPs in the distal humerus posterolateral column and shaft in a Chinese cadaveric study. They found that 75\% (33/44) of humeri were longer than $293.6 \mathrm{~mm}$, which is the cutoff value for plate protrusion in this study. However, they focused on mismatch at the shaft, not at the distal tip of the EADHP. Furthermore, plate-bone mismatch due to angulation of the shaft area can be resolved through plate bending, but distal plate protrusion is difficult to resolve, so it is necessary to predict whether the patient is an EADHP candidate before surgery.

EADHP anatomic fit mismatch should be predicted preoperatively to prevent implant removal and potential iatrogenic radial nerve damage. Trikha et al. [13] reported that approximately $59.5 \%(22 / 37)$ of patients treated using an EADHP exhibited prominence on the elbow posterolateral side. Among them, only one patient underwent implant removal. Although implant mismatch occurred, not all patients developed skin discomfort requiring implant removal. However, in the majority of cases, the EADHP is placed beneath the radial nerve in a posterior approach to distal humeral fractures, and no matter how cautious we are, iatrogenic radial nerve palsy can occur during implant removal. Thus, when skin protrusion due to EADHP anatomic fit mismatch at the distal tip and resulting discomfort are expected, alternative treatments should be considered.

As an alternative treatment, a lower profile plate for the distal medial tibia can be employed for distal humerus fractures through the same posterior approach [18]. This locking plate does not use the posterolateral column of the distal humerus and seems to be less affected by protrusion; however, whether the biomechanical properties of the plate are comparable to conventional EADHPs should be investigated. As another alternative, a locking compression plate used for the proximal humerus was suggested via an anterolateral approach $[19,20]$. A previous biomechanical study showed that modified use of a proximal humeral locking plate has comparable mechanical stability com- 
pared to EADHP [21]. Yin et al. [22] suggested both an anterolateral approach and a lateral approach to distal humeral extra-articular fractures as alternative surgical methods. Unlike EADHP, plate irritation was not reported in the clinical outcomes of the anterolateral approach or the lateral approach.

There are several limitations to this study. First, cadaveric studies have some important differences from in vivo studies. In this cadaveric research, the soft tissue was completely removed, but soft tissue dissection is limited during in vivo surgery. Second, the definition or threshold value for EADHP anatomic fit mismatch leading to skin protrusion and related discomfort was determined arbitrarily, because research on this topic is sparse. The threshold leading to protrusion and discomfort in patients may not be consistent with this value. Third, the sample size was small.

In conclusion, anatomic fit mismatch in distal humeral fractures after EADHP fixation has a negative linear correlation with humeral length and distal humeral width. In particular, for patients whose distal humeral width is less than $60.5 \mathrm{~mm}, 10 \mathrm{~mm}$ or greater plate protrusion is predicted when an EADHP is applied, and an alternative implant or approach should be considered during treatment planning.

\section{ORCID}

Joon-Ryul Lim

https://orcid.org/0000-0002-0123-7136

Tae-Hwan Yoon

https://orcid.org/0000-0002-2859-5240

Yong-Min Chun

https://orcid.org/0000-0002-8147-6136

\section{REFERENCES}

1. Court-Brown CM, Caesar B. Epidemiology of adult fractures: a review. Injury 2006;37:691-7.

2. Anglen J. Distal humerus fractures. J Am Acad Orthop Surg 2005;13:291-7.

3. Mighell MA, Stephens B, Stone GP, Cottrell BJ. Distal humerus fractures: open reduction internal fixation. Hand Clin 2015;31: 591-604.

4. Pollock JW, Faber KJ, Athwal GS. Distal humerus fractures. Orthop Clin North Am 2008;39:187-200.

5. Koonce RC, Baldini TH, Morgan SJ. Are conventional reconstruction plates equivalent to precontoured locking plates for distal humerus fracture fixation? A biomechanics cadaver study. Clin Biomech (Bristol, Avon) 2012;27:697-701.

6. Zalavras CG, Vercillo MT, Jun BJ, Otarodifard K, Itamura JM, Lee TQ. Biomechanical evaluation of parallel versus orthogonal plate fixation of intra-articular distal humerus fractures. J
Shoulder Elbow Surg 2011;20:12-20.

7. Stoffel K, Cunneen S, Morgan R, Nicholls R, Stachowiak G. Comparative stability of perpendicular versus parallel double-locking plating systems in osteoporotic comminuted distal humerus fractures. J Orthop Res 2008;26:778-84.

8. Illical EM, Farrell DJ, Siska PA, Evans AR, Gruen GS, Tarkin IS. Comparison of outcomes after triceps split versus sparing surgery for extra-articular distal humerus fractures. Injury 2014; 45:1545-8.

9. Meloy GM, Mormino MA, Siska PA, Tarkin IS. A paradigm shift in the surgical reconstruction of extra-articular distal humeral fractures: single-column plating. Injury 2013;44:1620-4.

10. Kharbanda Y, Tanwar YS, Srivastava V, Birla V, Rajput A, Pandit R. Retrospective analysis of extra-articular distal humerus shaft fractures treated with the use of pre-contoured lateral column metaphyseal LCP by triceps-sparing posterolateral approach. Strategies Trauma Limb Reconstr 2017;12:1-9.

11. Fawi H, Lewis J, Rao P, Parfitt D, Mohanty K, Ghandour A. Distal third humeri fractures treated using the SynthesTM 3.5-mm extra-articular distal humeral locking compression plate: clinical, radiographic and patient outcome scores. Shoulder Elbow 2015;7:104-9.

12. Capo JT, Debkowska MP, Liporace F, Beutel BG, Melamed E. Outcomes of distal humerus diaphyseal injuries fixed with a single-column anatomic plate. Int Orthop 2014;38:1037-43.

13. Trikha V, Agrawal P, Das S, Gaba S, Kumar A. Functional outcome of extra-articular distal humerus fracture fixation using a single locking plate: a retrospective study. J Orthop Surg (Hong Kong) 2017;25:2309499017727948.

14. Zhou Z, Tang Z, Zhao X, et al. Mismatch of AO anatomically shaped distal humeral plate with humeral shaft forward flexion angulation in adult Chinese population. Eur J Orthop Surg Traumatol 2014;24:1145-50.

15. Youden WJ. Index for rating diagnostic tests. Cancer 1950;3:325.

16. Ahmad M, Nanda R, Bajwa AS, Candal-Couto J, Green S, Hui AC. Biomechanical testing of the locking compression plate: when does the distance between bone and implant significantly reduce construct stability. Injury 2007;38:358-64.

17. Singhal S, Rao V. Estimation of total length of humerus from its segments. Med Sci Law 2011;51:18-20.

18. Yorukoglu AC, Demirkan AF, Buker N. Distal medial tibial locking plate for fixation of extraarticular distal humeral fractures; an alternative choice for fixation. Acta Orthop Traumatol Turc 2018;52:294-8.

19. Park JH, Kim JW, Oh CH, Choi KS, Hong JY, Kim JG. PHILOS plate osteosynthesis in metaphyseal fractures of the distal hu- 
merus through an anterolateral approach. Clin Shoulder Elbow 2015;18:128-32.

20. Sohn HS, Shin SJ. Modified use of a proximal humeral internal locking system (PHILOS) plate in extra-articular distal-third diaphyseal humeral fractures. Injury 2019;50:1300-5.

21. Lim JR, Yoon TH, Choi YR, Lee HM, Chun YM. Biomechanical evaluation of a modified proximal humeral locking plate appli- cation for distal extra-articular diaphyseal humeral fractures. J Orthop Res 2020 Nov 22 [Epub]. https://doi.org/10.1002/jor. 24925.

22. Yin P, Zhang L, Mao Z, et al. Comparison of lateral and posterior surgical approach in management of extra-articular distal humeral shaft fractures. Injury 2014;45:1121-5. 